\section{(1)}

CrossMark

\title{
Effects of sleep apnoea therapy on blood pressure and metabolism: a CPAP sex gap?
}

\author{
Frédéric Gagnadoux ${ }^{1,2,3}$, Pascaline Priou ${ }^{1,2,3}$, Nicole Meslier $^{1,2,3}$ and \\ Wojciech Trzepizur ${ }^{1,2,3}$
}

Affiliations: ${ }^{1}$ Université Bretagne Loire, Angers, France. ${ }^{2}$ Département de Pneumologie, CHU, Angers, France. ${ }^{3}$ INSERM UMR 1063, Angers, France.

Correspondence: Frédéric Gagnadoux, Université d'Angers, CHU Angers, Département de Pneumologie, 4 rue Larrey, 49033 Angers Cedex, France. E-mail: frgagnadoux@chu-angers.fr

@ERSpublications

CPAP therapy reduces blood pressure but has no impact on metabolic profile in women with sleep apnoea http://ow.ly/AAKj30cQb6X

Cite this article as: Gagnadoux F, Priou P, Meslier N, et al. Effects of sleep apnoea therapy on blood pressure and metabolism: a CPAP sex gap? Eur Respir J 2017; 50: 1700987 [https://doi.org/10.1183/ 13993003.00987-2017].

Obstructive sleep apnoea (OSA) is a highly prevalent disease characterised by recurrent episodes of complete or partial upper airway obstruction during sleep, leading to intermittent hypoxia and sleep fragmentation. Most recent estimates of OSA prevalence suggest that $13 \%$ of men and $6 \%$ of women have clinically significant OSA $[1,2]$. There is evidence from population-based and clinic-based cohort studies that moderate-to-severe OSA is independently associated with an increased incidence of cardiovascular events $[3,4]$. Besides OSA-related variables, comorbid conditions including hypertension and metabolic dysfunction play an important role in the development of cardiovascular diseases in OSA patients [4]. In the clinical setting, it has been estimated that at least half of patients with OSA fulfil the criteria for the metabolic syndrome [5]. Furthermore, the prevalence of metabolic syndrome increases with OSA severity $[5,6]$. An independent dose-response relationship has been demonstrated between OSA severity and the features of metabolic syndrome, including visceral adiposity [7], hypertension [8], metabolic dyslipidaemia [6] and impaired glucose metabolism [9, 10]. Considering cardiovascular prevention as a therapeutic objective in OSA patients, it is crucial to determine whether OSA therapy can lead to clinically relevant improvements in blood pressure, and glucose and lipid metabolism.

Most previous randomised controlled trials (RCTs) evaluating the impact of continuous positive airway pressure (CPAP) therapy on health outcomes were performed in unselected, predominantly male OSA patients. It is increasingly recognised that there is a between-sex difference in the clinical manifestations of OSA, with women more frequently experiencing symptoms of depression and anxiety, and less frequently complaining of excessive daytime sleepiness [11-13]. Sex differences have also been observed in cardiovascular outcomes, with more severe endothelial dysfunction [14], and higher risks of coronary heart disease and stroke in women [15]. Epidemiological data have shown that OSA in females is related to obesity, hypertension and diabetes but not to daytime sleepiness [11]. Altogether, these findings highlight the need for adequately powered RCTs examining sex differences in CPAP treatment response with particular focus on blood pressure and metabolic profile. 
In this issue of the European Respiratory Journal, CAMPOS-RODRIGUEZ et al. [16] address this important concern in a multicentre, open-label RCT examining the effect of CPAP on blood pressure, glucose and lipid profile in 307 women diagnosed with moderate-to-severe OSA. Among included patients, $31 \%$ satisfied the hypertension criteria at baseline and 55\% were on antihypertensive medication. The study has a number of important strengths including a large sample size, a multicentre design, the use of fixed pressure CPAP (which has been found to be more effective than auto-CPAP in reducing $24 \mathrm{~h}$ diastolic BP [17]) and a relatively high rate of CPAP compliance with $75 \%$ of participants using the device for at least $4 \mathrm{~h}$ per night. The main limitation of the study is the use of office rather than 24 -h ambulatory blood pressure monitoring, which is considered the reference standard for the diagnosis of hypertension, particularly in OSA patients in whom hypertension is predominantly nocturnal [8].

The first finding of this study was that 12 weeks of CPAP therapy was associated with a modest but significant reduction in diastolic blood pressure $(-2.04 \mathrm{mmHg}, 95 \% \mathrm{CI}-4.02--0.05 \mathrm{mmHg})$ compared to controls [16]. This finding is highly consistent with most RCTs and meta-analyses reporting drops of 2-2.5 $\mathrm{mmHg}$ and $1.5-2 \mathrm{mmHg}$ in systolic and diastolic blood pressure, respectively, in unselected OSA populations [18]. In line with previous reports [19], several characteristics at baseline were associated with a greater blood pressure reduction on CPAP including severe OSA, and higher baseline blood pressure and excessive daytime sleepiness. Improving blood pressure control in hypertensive patients is a major concern as lowering blood pressure reduces cardiovascular risk in a dose-dependent manner [20]. In the SAVE (Sleep Apnea CardioVascular Endpoints) trial, CPAP therapy averaging $3.3 \mathrm{~h}$ per night had no significant impact on blood pressure, and did not prevent cardiovascular events in patients with moderate-to-severe OSA and established cardiovascular disease [21]. Although the minimum threshold of CPAP adherence needed to obtain a clinically significant blood pressure reduction is unknown, improving CPAP compliance may contribute to improving blood pressure control, especially by avoiding residual rapid eye movement-related sleep disordered breathing at the end of the night [22]. The superiority of fixed pressure CPAP over auto-CPAP in reducing 24-h blood pressure might also be of particular interest in OSA patients with poorly controlled or resistant hypertension [17]. Considering the multifactorial pathophysiology of OSA-associated hypertension, the combination of CPAP therapy, antihypertensive drugs and weight-loss interventions appears to be the most promising strategy to improve blood pressure control in OSA patients [18]. Although antihypertensive drugs such as valsartan have been found to be markedly more effective than CPAP on OSA-associated hypertension [23], the combination of blood pressure-lowering drugs and CPAP has additive effects on blood pressure $[23,24]$. As randomised trials have shown the beneficial effects of weight loss on blood pressure [25], weight-loss intervention may also be a major component of the strategies used to improve blood pressure control in obese patients with OSA. In a recent randomised trial including 146 patients with obesity and moderate-to-severe OSA, adherence to a regimen of weight loss and CPAP resulted in incremental reductions in blood pressure compared with either intervention alone [26].

The second finding of this study is that CPAP therapy for 3 months had no impact on metabolic profile in women with moderate-to-severe OSA [16]. As acknowledged by the authors, most women had baseline glucose and lipid values within the normal range, which may have been associated with a floor effect of the intervention. However, the present finding is in accordance with most recent RCTs and meta-analyses. A systematic review of RCTs concluded that in unselected OSA patients, it is unrealistic to expect a clinically relevant improvement in metabolic and inflammatory markers with CPAP therapy [27]. In a recent RCT, CPAP therapy alone had remarkably limited effects on metabolic dysfunction in obese patients with OSA whereas weight loss provided an incremental reduction in insulin resistance and serum triglyceride levels when combined with CPAP [26]. Whether CPAP therapy has the power to improve glucose metabolism is still debated. In OSA patients with pre-diabetes or overt type 2 diabetes, laboratory-based RCTs found that short-term CPAP therapy with high levels of compliance may improve glucose control $[28,29]$. However, these findings were not confirmed by a large multicentre RCT, which showed that 6 months of CPAP therapy had no effect on glycated haemoglobin in diabetic patients with moderate-to-severe OSA [30].

The study by CAmpos-Rodriguez et al. [16] provides no strong argument in support of between-sex differences in the effect of CPAP on blood pressure and metabolism. In line with previous data in unselected OSA populations, the present findings highlight the need for a multidisciplinary approach to manage OSA patients with cardiometabolic comorbidities [27]. Further studies are required to evaluate the effects of CPAP therapy on cardiometabolic health in the context of weight-loss interventions for OSA patients with metabolic disorders. Personalised medicine is an emerging concept taking into account the marked heterogeneity of OSA in terms of structural and physiological risk factors, clinical phenotypes, molecular signatures, health outcomes, and treatment response [12, 31, 32]. A recent report demonstrated that blood pressure response to CPAP in OSA patients with resistant hypertension can be predicted by a cluster of three specific plasma microribonucleic acids [33]. Further studies are needed to identify metabolic phenotypes that are more likely to be responsive to CPAP therapy. 


\section{References}

1 Heinzer R, Vat S, Marques-Vidal P, et al. Prevalence of sleep-disordered breathing in the general population: the HypnoLaus study. Lancet Respir Med 2015; 3: 310-318.

2 Peppard PE, Young T, Barnet JH, et al. Increased prevalence of sleep-disordered breathing in adults. Am J Epidemiol 2013; 177: 1006-1014.

3 Dong JY, Zhang YH, Qin LQ. Obstructive sleep apnea and cardiovascular risk: meta-analysis of prospective cohort studies. Atherosclerosis 2013; 229: 489-495.

4 Kendzerska T, Gershon AS, Hawker G, et al. Obstructive sleep apnea and risk of cardiovascular events and all-cause mortality: a decade-long historical cohort study. PLoS Med 2014; 11: e1001599.

5 Bonsignore MR, Esquinas C, Barcelo A, et al. Metabolic syndrome, insulin resistance and sleepiness in real-life obstructive sleep apnoea. Eur Respir J 2012; 39: 1136-1143.

6 Trzepizur W, Le Vaillant M, Meslier N, et al. Independent association between nocturnal intermittent hypoxemia and metabolic dyslipidemia. Chest 2013; 143: 1584-1589.

7 Vgontzas AN, Papanicolaou DA, Bixler EO, et al. Sleep apnea and daytime sleepiness and fatigue: relation to visceral obesity, insulin resistance, and hypercytokinemia. J Clin Endocrinol Metab 2000; 85: 1151-1158.

8 Parati G, Lombardi C, Hedner J, et al. Recommendations for the management of patients with obstructive sleep apnoea and hypertension. Eur Respir J 2013; 41: 523-538.

9 Anothaisintawee T, Reutrakul S, Van Cauter E, et al. Sleep disturbances compared to traditional risk factors for diabetes development: Systematic review and meta-analysis. Sleep Med Rev 2016; 30: 11-24.

10 Priou P, Le Vaillant M, Meslier N, et al. Independent association between obstructive sleep apnea severity and glycated hemoglobin in adults without diabetes. Diabetes Care 2012; 35: 1902-1906.

11 Franklin KA, Sahlin C, Stenlund H, et al. Sleep apnoea is a common occurrence in females. Eur Respir J 2013; 41: 610-615.

12 Gagnadoux F, Le Vaillant M, Paris A, et al. Relationship between OSA clinical phenotypes and CPAP treatment outcomes. Chest 2016; 149: 288-290.

13 Ye L, Pien GW, Weaver TE. Gender differences in the clinical manifestation of obstructive sleep apnea. Sleep Med 2009; 10: 1075-1084

14 Faulx MD, Larkin EK, Hoit BD, et al. Sex influences endothelial function in sleep-disordered breathing. Sleep 2004; 27: 1113-1120.

15 Campos-Rodriguez F, Martinez-Garcia MA, Reyes-Nunez N, et al. Role of sleep apnea and continuous positive airway pressure therapy in the incidence of stroke or coronary heart disease in women. Am J Respir Crit Care Med 2014; 189: 1544-1550.

16 Campos-Rodriguez F, Gonzalez-Martinez M, Sanchez-Armengol A, et al. Effect of continuous positive airway pressure on blood pressure and metabolic profile in women with sleep apnoea. Eur Respir J 2017; 50: 1700257.

17 Pepin JL, Tamisier R, Baguet JP, et al. Fixed-pressure CPAP versus auto-adjusting CPAP: comparison of efficacy on blood pressure in obstructive sleep apnoea, a randomised clinical trial. Thorax 2016; 71: 726-733.

18 Javaheri S, Barbe F, Campos-Rodriguez F, et al. Sleep apnea: types, mechanisms, and clinical cardiovascular consequences. J Am Coll Cardiol 2017; 69: 841-858.

$19 \mathrm{Hu} \mathrm{X}$, Fan J, Chen S, et al. The role of continuous positive airway pressure in blood pressure control for patients with obstructive sleep apnea and hypertension: a meta-analysis of randomized controlled trials. J Clin Hypertens (Greenwich) 2015; 17: 215-222.

20 Ettehad D, Emdin CA, Kiran A, et al. Blood pressure lowering for prevention of cardiovascular disease and death: a systematic review and meta-analysis. Lancet 2016; 387: 957-967.

21 McEvoy RD, Antic NA, Heeley E, et al. CPAP for prevention of cardiovascular events in obstructive sleep apnea. N Engl J Med 2016; 375: 919-931.

22 Mokhlesi B, Finn LA, Hagen EW, et al. Obstructive sleep apnea during REM sleep and hypertension. Results of the Wisconsin Sleep Cohort. Am J Respir Crit Care Med 2014; 190: 1158-1167.

23 Pepin JL, Tamisier R, Barone-Rochette G, et al. Comparison of continuous positive airway pressure and valsartan in hypertensive patients with sleep apnea. Am J Respir Crit Care Med 2010; 182: 954-960.

24 Thunstrom E, Manhem K, Rosengren A, et al. Blood pressure response to losartan and continuous positive airway pressure in hypertension and obstructive sleep apnea. Am J Respir Crit Care Med 2016; 193: 310-320.

25 Horvath K, Jeitler K, Siering U, et al. Long-term effects of weight-reducing interventions in hypertensive patients: systematic review and meta-analysis. Arch Intern Med 2008; 168: 571-580.

26 Chirinos JA, Gurubhagavatula I, Teff K, et al. CPAP, weight loss, or both for obstructive sleep apnea. $N$ Engl J Med 2014; 370: 2265-2275.

27 Jullian-Desayes I, Joyeux-Faure M, Tamisier R, et al. Impact of obstructive sleep apnea treatment by continuous positive airway pressure on cardiometabolic biomarkers: a systematic review from sham CPAP randomized controlled trials. Sleep Med Rev 2015; 21: 23-38.

28 Mokhlesi B, Grimaldi D, Beccuti G, et al. Effect of one week of 8-hour nightly continuous positive airway pressure treatment of obstructive sleep apnea on glycemic control in type 2 diabetes: a proof-of-concept study. Am J Respir Crit Care Med 2016; 194: 516-519.

29 Mokhlesi B, Grimaldi D, Beccuti G, et al. Effect of one week of CPAP treatment of obstructive sleep apnoea on 24-hour profiles of glucose, insulin and counter-regulatory hormones in type 2 diabetes. Diabetes Obes Metab 2017; 19: 452-456.

30 Shaw JE, Punjabi NM, Naughton MT, et al. The effect of treatment of obstructive sleep apnea on glycemic control in type 2 diabetes. Am J Respir Crit Care Med 2016; 194: 486-492.

31 Ye L, Pien GW, Ratcliffe SJ, et al. The different clinical faces of obstructive sleep apnoea: a cluster analysis. Eur Respir J 2014; 44: 1600-1607.

32 Zinchuk AV, Gentry MJ, Concato J, et al. Phenotypes in obstructive sleep apnea: A definition, examples and evolution of approaches. Sleep Med Rev 2016 [in press https://doi.org/10.1016/j.smrv.2016.10.002].

33 Sanchez-de-la-Torre M, Khalyfa A, Sanchez-de-la-Torre A, et al. Precision medicine in patients with resistant hypertension and obstructive sleep apnea: blood pressure response to continuous positive airway pressure treatment. J Am Coll Cardiol 2015; 66: 1023-1032. 